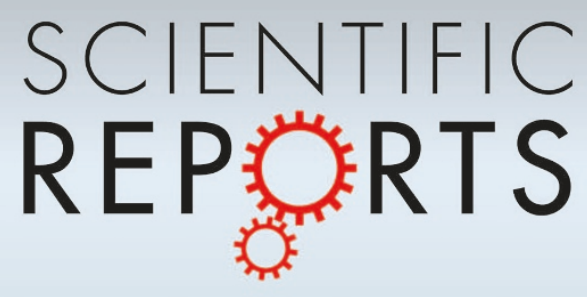

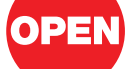

SUBJECT AREAS: APPLIED MATHEMATICS

APPLIED PHYSICS

COMPUTATIONAL SCIENCE

STATISTICAL PHYSICS, THERMODYNAMICS AND NONLINEAR DYNAMICS

Received

11 December 2012

Accepted

8 February 2013

Published

9 April 2013

Correspondence and requests for materials should be addressed to G.C. Iguido. caldarelli@imtlucca.it)

\section{Evolution of Controllability in Interbank Networks}

\author{
Danilo Delpini 1,2, Stefano Battiston ${ }^{3}$, Massimo Riccaboni ${ }^{2,4}$, Giampaolo Gabbi ${ }^{5,6}$, Fabio Pammolli ${ }^{2,7}$ \\ \& Guido Caldarelli2,8,9,10
}

${ }^{1}$ Department of Economics and Business - DiSEA, University of Sassari, via Muroni 25, 07100 Sassari, Italy, ${ }^{2}$ IMT Institute for Advanced Studies, Piazza San Ponziano 6, 55100 Lucca, Italy, ${ }^{3}$ Chair of Systems Design, MTEC, ETH Zurich, Weinbergstrasse 5658, 8092 Zurich, Switzerland, ${ }^{4}$ Department of Managerial Economics, Strategy and Innovation, Katholieke Universiteit Leuven, 3000 Leuven, Belgium, ${ }^{5}$ University of Siena, Department of Management and Law, Piazza S. Francesco, 53100 Siena, Italy, ${ }^{6}$ SDA Bocconi School of Management, via Bocconi 8, 20136 Milan, Italy, ${ }^{7}$ Center for Polymer Studies and Department of Physics, Boston University Boston, MA $02115,{ }^{8}$ Istituto dei Sistemi Complessi (ISC), Department of Physics, Università "Sapienza", PI. Aldo Moro 2, 00185 Roma, Italy, 'London Institute for Mathematical Sciences, 35a South St. Mayfair W1K 2XF London UK, ${ }^{10}$ Linkalab, Complex Systems Computational Laboratory, 09129 Cagliari, Italy.

The Statistical Physics of Complex Networks has recently provided new theoretical tools for policy makers. Here we extend the notion of network controllability to detect the financial institutions, i.e. the drivers, that are most crucial to the functioning of an interbank market. The system we investigate is a paradigmatic case study for complex networks since it undergoes dramatic structural changes over time and links among nodes can be observed at several time scales. We find a scale-free decay of the fraction of drivers with increasing time resolution, implying that policies have to be adjusted to the time scales in order to be effective. Moreover, drivers are often not the most highly connected "hub" institutions, nor the largest lenders, contrary to the results of other studies. Our findings contribute quantitative indicators which can support regulators in developing more effective supervision and intervention policies.

he recent financial crisis has shown how distress or disfunction in the banking sector eventually engender serious repercussions in both the real economy and society at large. It has been increasingly recognized that (a portion of) the banking sector can be regarded as a complex network ${ }^{1-3}$, and the same also holds for the market investment network, i.e. the network of links among the financial agents playing the role of buyers/sellers, brokers, dealers, market makers within securities, derivatives or insurance markets ${ }^{4}$. Substantial, heterogeneous uncertainty is due to the lack of complete information about mutual financial positions. Statistical Physics allows to focus on the structure of the mutual relationships among institutions inferred from recorded transactions. Accordingly, Statistical Physics potentially has much to contribute to the characterization, especially at a systemic level, of properties such as contagion, robustness and resilience. A large body of work has focused on the issue of financial complexity ${ }^{5}$ and complex financial networks ${ }^{2,6-10}$.

The system we study is an example of a temporal network, a time ordered sequence of realizations of the same system represented as a graph. These systems are frequently studied by collecting all nodes and edges over a time interval $\Delta t$, and recent contributions, see ${ }^{11}$, address the issue of quantifying the consequences of this aggregation procedure in the description of dynamical processes within the network. However, to our knowledge, the role of $\Delta t$ in defining to what extent a network's state can be driven by means of external controllers, as well as the way how controllability varies during the network's evolution have yet to be studied.

Here we fill this gap by applying the new concept of network controllability to the network of interbank loans. This network represents a building block of modern financial economies, also representing a crucial gear for the transmission of monetary policies to the real economy. We systematically investigate its controllability over a time span $(\approx 10$ years), which is very long with respect to the time scale of the system's dynamics $(\approx$ days $)$, at different levels of time aggregation. Indeed, during the time span of the data the interbank lending system undergoes dramatic changes in its functioning. From 1999 the network shrinks steadily by $50 \%$ in size; lending volumes increase and then, between 2007 and 2009, drop abruptly by 80\% (see Fig. S1 in Supplementary Information, Section I. A). Finally, we shed some light on the relationship between controllability and link aggregation time-scale. A common feature of many empirical networks is indeed that interaction between nodes occurs at discrete points in time, while the network structure is observed after aggregating these interactions into 
weighted links ${ }^{12}$ over a certain time window. Depending on the time scale, the resulting network structure may vary greatly, and so may our ability to control the system.

In the case of interbank deposit contracts we have ties which can potentially propagate defaults or distress ${ }^{13-16}$. A first important debate in financial regulation concerns how to identify and deal with the so-called too-big-too-fail (TBTF) institutions ${ }^{17}$. This notion has recently been extended over to a network context as a "too-centralto-fail" specification ${ }^{2}$, and it deals with the propagation of potential losses due to the exposures of any given bank to its counterparties. However, the efficiency of the financial system and more in general its well-functioning crucially depends on the willingness of banks to lend to each other. This is influenced by the liquidity availability of the lender as well as by the perceived risk that the counterpart will be illiquid and/or insolvent at the expiry date. Both of these aspects may be a vehicle of contagion and are sources of systemic risk. Nevertheless, difficult access to funding for a bank can signal both lack of liquidity in the system and enhanced counterpart risk as perceived by lenders. As a consequence, the same bank is likely to reduce its funding to others in the immediately following period. Uncertainty on the future value of counterparties' assets disrupts trust among banks leading to a contagion of funding withdrawals and eventually to a dry-up of credit from banks to real-economy firms ${ }^{18-20}$. Therefore, another debate, which is currently receiving a great deal of attention, regards the number of institutions that should be the object of supervision and liquidity provisions from their authorities: only a few big ones, according to some; all of them according to others. We contribute to these concerns with an analysis of these systems employing the tools provided to us by Statistical Physics.

In particular, we study the time evolution of the controllability for the interbank lending network and we put it forth as a new method to help identify the institutions that should be supervised. Our approach considers the interbank market as a complex network where lending volumes flow from one node to another based on market rules and individual incentive. We are thereby able to translate the problem of ensuring the good functioning of the market onto the problem of identifying the subset of nodes that drive the dynamics.

In this paper we refer explicitly to the notion of network controllability developed $\mathrm{in}^{21}$ building on the concept of structural controll$a_{b i l i t y}^{22}$. This notion deals with the problem of driving a network's state toward a desired target by the application of external controllers to some nodes. By network state we mean the vector of the states $\left(x_{1}(t), x_{2}(t), \ldots, x_{N}(t)\right)$ of all the nodes and by "controller" any (timedependent) action which modifies the state of the node to which it applies. Without controllers, a node's state will also vary proportionally according to the influence of its neighbours. The minimal set of nodes to control (drivers), in order to bring the network to an arbitrary final state, is found by solving a geometric problem: given a maximum matching of the network's graph, the drivers are the nodes that remain unmatched (see Supplementary Information, Section II. A).

\section{Results}

Trust evaporation and liquidity hoarding are major causes of gridlocks and inefficient behaviour in interbank lending systems during periods of financial distress. In these circumstances, liquidity funding becomes difficult and the transmission of interest rate decisions from central banks to the interbank network breaks.

We identify the relevant state variable $x_{i}$ as the level of funding bank $i$ provides to the others and assume that, in turn, $x_{i}$ depends on the funding it gets from its neighbours. In other words, we assume that banks influence each others through "funding contagion" and that the influence of bank $i$ on $j$ is somewhat proportional to the funding provided by $i$ to $j$ (more details in Supplementary Information, Section II.B).

In this context, the concept of external "control" is implemented by liquidity interventions of central banks in individual institutions of the network. These actions can involve liquidity refinancing or, in principle, dedicated credit facilities. Central banks cannot actually enforce banks to lend, but they can provide liquidity to key-role banks in the market on a much larger basis than they need. Therefore, very liquid banks are de facto induced to provide liquidity to the other players. This mechanism is effective in the eurosystem and in the UK, where central banks often provide liquidity to large institutions, in order to allow the rest of the system to receive liquidity. Here we suggest that the effectiveness of these practices may be enhanced by concentrating them on the network's drivers.

We analyse the controllability of interbank money markets empirically, focusing on the specific case of the Italian electronic trading system (e-MID), which is open to European banking players, and for which a time series of micro data is available. Following the network evolution over time we detect the banks that are more relevant from a control perspective. For them, we analyse the changes of the relevant topological and financial quantities, then clarifying the role played by drivers in this system. Finally, we address the resilience of the network drivers, that is the correlation between driver sets at different times.

Generally, daily transactions are rather volatile and, in order to reduce the level of the fluctuations, we can aggregate them over different time horizons, denoted by $\Delta$. The resulting aggregated networks may well have different properties than the daily graph. Defining an optimal scale a priori is not straightforward, but the liquidity coverage ratio requirement, imposed by the Basel Committee (Basel III) proposal, points to the monthly scale as a reasonable time window: it forces banks to maintain a liquidity buffer of high quality, liquid assets, to cope with potential liquidity outflows during the following 30 days. For the first time we study how the controllability properties of the network depend on the observation time scale. We find a high degree of dependence for both the set of controlling banks and their impact with respect to the total volume of lending in the system.

We adopt the fraction of network drivers as an indicator of control efficiency and robustness ${ }^{23,24}$. The first result is that the observation of the network on a daily scale may lead to the misleading conclusion that the system controllability is inherently low. This is because more than half of the banks are drivers. However, our study reveals that the fraction of drivers decays according to a power law as the aggregation scale gets larger. Therefore, on a monthly basis the system proves to be quite efficient from the control perspective. A similar bias is also found when looking at the share of lending for the drivers. The latter increased remarkably during the recent years of financial turmoil, signalling a concentration of credit at those sites which actually control the network. We highlight that the banks that drive the lending system are not the hubs, nor do they correspond to the top lenders as could be expected. Finally, we show that indicators like the stability of the drivers set over time or their closeness centrality point to the monthly scale as the natural scale for observing the system.

At every aggregation scale $\Delta$ and for every available network instance, we compute the maximum matching of the graph, and thereby we identify the set of driver nodes (Fig. 1 (a) shows a daily network instance, with the driver nodes highlighted). The number of maximum matchings for directed graphs of this size can be rather large, and enumerating all of them is an intensive numerical task. Here we assume that a notion of cost can be attached to network control, and that this cost is inversely proportional to the sum of weights of the maximum matching edges. Therefore, in the following analysis, we always select the maximum matching of maximum weight (the weight being given by the sum of edge weights in the matching). We don't provide a general proof supporting our 
(a)

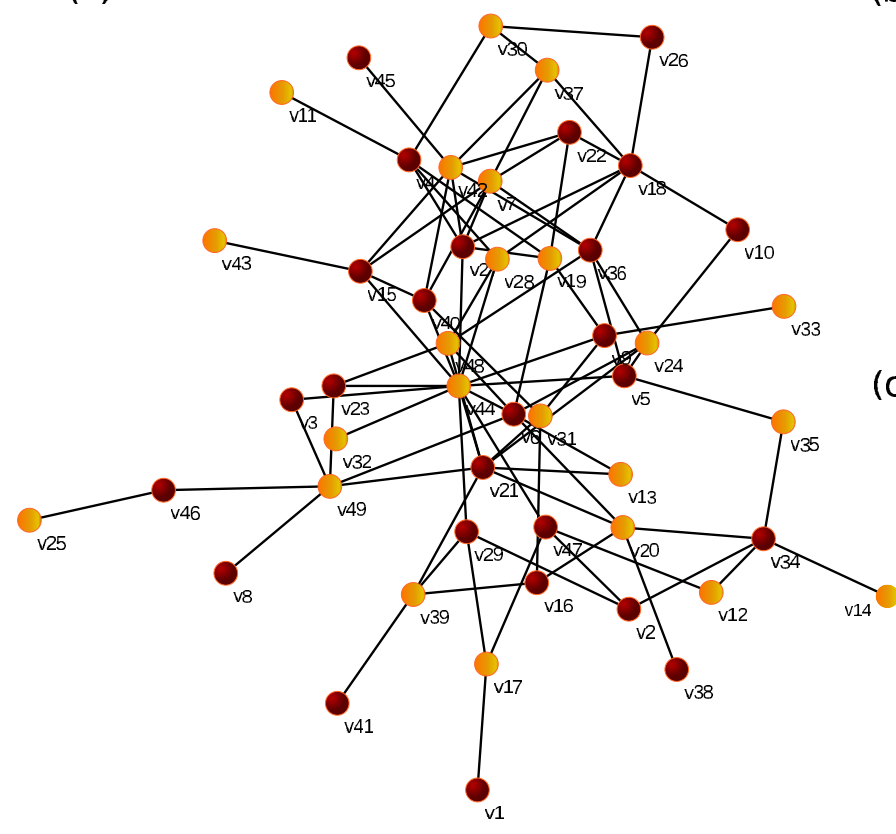

(b)

(c)

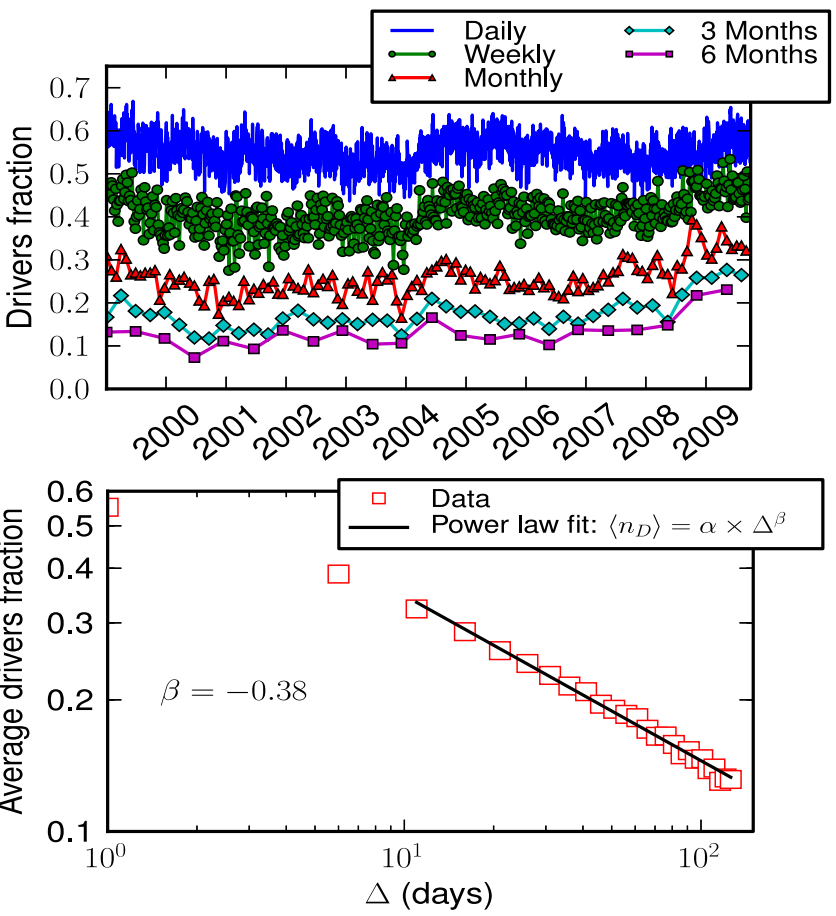

Figure $1 \mid$ (a). A sample snapshot of the daily interbank lending network. External inputs on the yellow nodes (drivers) allow to control the state of the whole system. (b). Time evolution of the fraction of drivers: at the monthly scale less than $40 \%$ of the banks drive the system. (c). The average fraction of drivers decays with a neat power law scaling with the aggregation scale $\Delta$.

assumption and, certainly, different choices are possible. Nevertheless, it can be supported by heuristic arguments like those discussed in Supplementary Information, Section II.A. In particular, from an economic point of view, we expect "funding contagion" to take place mostly through channels characterized by strong credit relations (weights).

In Fig. 1 (b) we show the evolution of the fraction $n_{D}$ of unmatched vertices over the considered years at the different levels of aggregation, $\Delta$. Overall, this controllability parameter is fairly stable, with relative variations below $10 \%$. At the daily scale, controlling nodes always take a large fraction ranging from about $52 \%$ to $60 \%$. This would be interpreted as a critical point: if the central bank intervened, more than one half of the banks should be monitored by regulators. However, the $n_{D}$ values, on average, decrease monotonically as a function of the aggregation scale and the monthly network appears to be controlled by less than $30 \%$ of the banks. Such a value of $n_{D}$ supports the possibility for the regulator to implement targeted interventions in just the driver banks, and differentiates this system from other networks like the WWW or the Internet studied in $^{21}$ where
$n_{D}>50 \%$. Starting in 2008 we also notice a raising trend in $n_{D}$ for all values of $\Delta$. Thus a qualitative correspondence exists with the credit crisis triggered by subprime mortgages (Lehman-Brothers bankruptcy). To gain deeper insight in the scaling of the driver fraction with the aggregation scale, in Fig. 1 (c) we look at the average value $\left\langle n_{D}\right\rangle$ for values of $\Delta$ ranging from 1 day to 126 days. For the system at hand, we find a neat power law decay with scaling exponent $\beta=0.38$. This finding implies there is no characteristic time scale for the fraction of drivers in the system. It is not possible to select an optimal aggregation time based only on $n_{D}$, and we will see in the following section that other quantities give clearer indications in this regard. The value we find for the scaling exponent also means that $n_{D}$ decreases less than proportionally with $\Delta$, so that $\left\langle n_{D}\right\rangle$ reduces roughly from 0.25 to 0.15 when passing from $\Delta=1$ month to $\Delta=6$ months. Different levels of aggregation correspond to networks with different connectivity, which requires different control strategies. Different scales could serve different supervision purposes and policy makers could adopt the instruments that are more effective for the time horizon of interest for the control. (a)

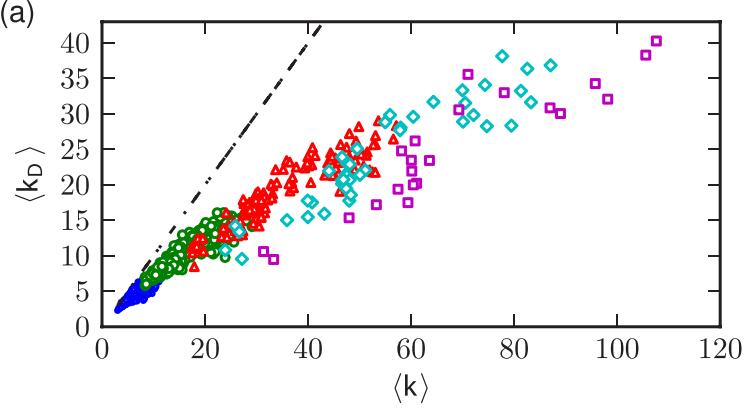

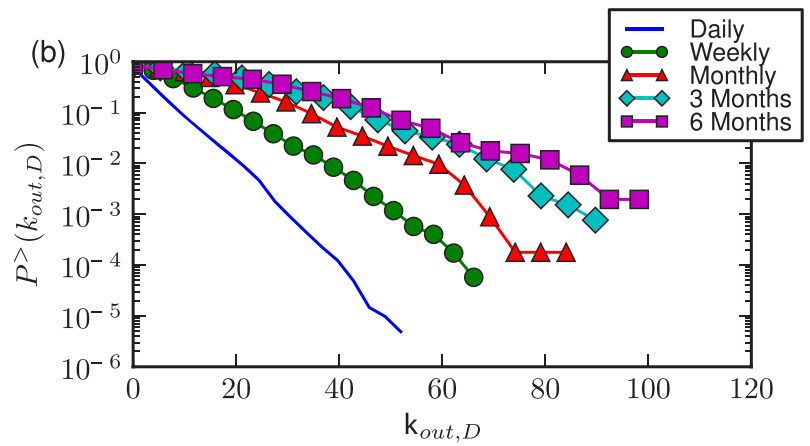

Figure $2 \mid$ (a). Scatter plot of the mean degree of driver vertices versus the mean degree in the network. On average drivers do not correspond to hubs. (b). Complementary of the cumulative distribution function for the out-degree of drivers nodes. Even though the network is scale free, the out-degree of drivers decays faster than a power law. 


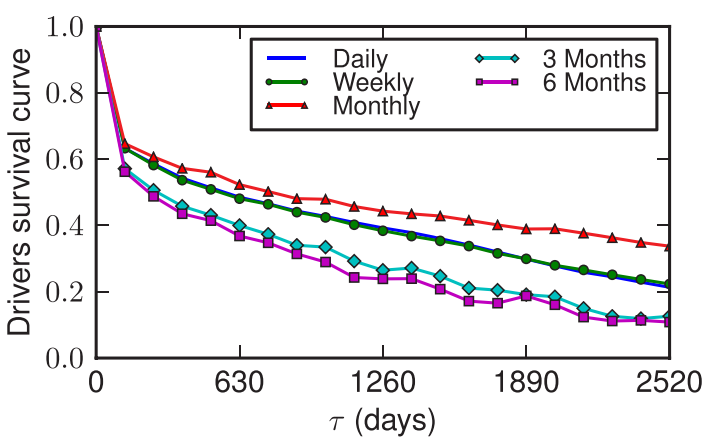

Figure 3 The slow decay of the driver resilience; this long-range memory effect makes control sets rather stable with respect to the network time change. The highest stability is achieved at the monthly scale.

Power law scalings have been repeatedly observed in economics and finance $\mathrm{e}^{5,25-27}$; here it emerges as a specific aspect of the system controllability resulting from the process of aggregating transactions across successive time periods. Generally, we expect $n_{D}$ to decrease with the average degree $\langle k\rangle$, with a minimum value of $1 / N$ for a complete (or a strongly connected) graph, where $N$ stands for the number of nodes. Actually, the process of aggregation leads to denser networks and the scaling of $n_{D}$ with $\Delta$ may be regarded as a mechanic effect. Nevertheless, the power-law profile of this scaling is peculiar to the system at hand (see the relative discussion in Supplementary Information, Section II.B).

To get a feeling for the role the drivers play in the network, in Fig. 2 (a) we plot their average degree $\left\langle k_{D}\right\rangle$ as a function of the average degree in the network $\langle k\rangle$ and we find $\left\langle k_{D}\right\rangle\langle\langle k\rangle$ systematically. This evidence (discussed in $^{21}$ for several biological, technological and social networks) clarifies that in the bank network the drivers are not the hubs, regardless of the aggregation level, and hubs are controlled only indirectly. Therefore, every "too connected to fail" strategy must be carefully considered before any bailing-out procedure.

Usually, driver banks affect their neighbours while not being affected; correspondingly their average in-degree can be shown to approximate zero on average. In Fig. 2 (b) we plot the complementary of the empirical cumulative distribution function $P^{>}\left(k_{\text {out }, D}\right)$ of their out-degree. It is not power-law as it would be expected from past analyses ${ }^{8}$. This finding is somewhat expected from Fig. 2 (a): for the most part, drivers are not hubs, so the probability of them having high degree must decay faster than for the whole set of nodes.

The control set of drivers will change over time. However, inspection of the drivers resilience (see Eq. (2) in Supplementary Information, Section II. C) from Fig. 3 shows that the system is characterized by long-range memory. The survival function has a very slow, almost linear decay, and after six months nearly $60 \%$ of drivers are still in the control set. Surprisingly, the level of the curve does not follow a monotonic trend with $\Delta$ : the control configuration is maximally stable at the monthly scale for which $r_{D}$ exhibits the slowest decay. This result supports the proposal of the Basel III Committee to introduce a 30-day liquidity coverage ratio, and suggests the monthly scale as a reasonable time window for observing the system.

The special role of this time horizon for the observation of the system properties is also confirmed by the drivers average closeness represented by the red bars in Fig. 4 (a). The closeness quantifies the centrality of drivers with respect to the lending relationships to direct and indirect borrowers (see Supplementary Information, Section I. C). As for the case of the resilience, we find here a non-trivial behaviour of the curve when varying the aggregation time scale: the closeness centrality is maximized at the monthly scale.

It is of major importance to investigate the "size" of the controlling institutions in terms of exchanged volumes. The yellow bars in Fig. 4 (a) show the fraction $v_{D}$ of the aggregate lending volume which is accounted for by the driver nodes. Even in this case, we find a crucial dependence on the aggregation scale. On average, the drivers of the daily network account for $70 \%$ of the total volume. This percentage largely decreases when we look at the system across a wider time period, and at the monthly level, the drivers of the system are responsible for only less than $30 \%$ of the total lending.

A relevant question is whether drivers correspond to the top lenders of the credit network. We addressed this issue by sorting banks in descending order of lending volume and defining the "top lenders" as the first $m_{\text {top }}$ banks in the list whose lending approximates (and is not larger than) $80 \%$ of the total lending provided in that time period. We give the name $B_{t o p}$ to the set of top lenders and, for all network instances we compute the overlap $n_{D, \text { top }}=\left|D \cap B_{\text {top }}\right| /\left|B_{\text {top }}\right|$; if drivers were just the top lenders, we would expect $n_{D \text {,top }} \approx 1$. The time evolution of $n_{D \text {, top }}$, for the different aggregation scales, is plotted in Fig. 4 (b), showing that the extent of the overlap strongly depends on $\Delta$. More interestingly, we also found that searching for drivers does not merely reduce down to detecting top lender banks: before 2008 , at the monthly scale less than $40 \%$ of the leading banks play a role in system control. After 2008, at all time scales, a fast rise is observed in $n_{D \text {, top }}$; we conclude that the liquidity shortening observed during the crisis (see Supplementary Information, Section I.A) comes with an increase of the centrality of controlling banks, as measured by their weighted out-degree, inside the lending system.

\section{Discussion}

The recent financial crisis has been forcing central banks to implement aggressive and creative policy actions. Radically new strategies (a)

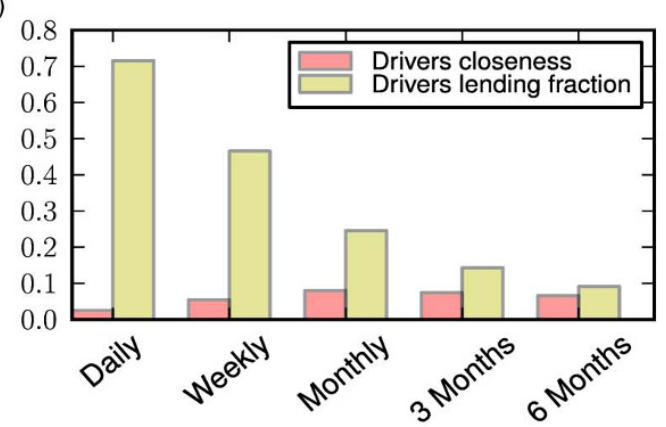

(b)

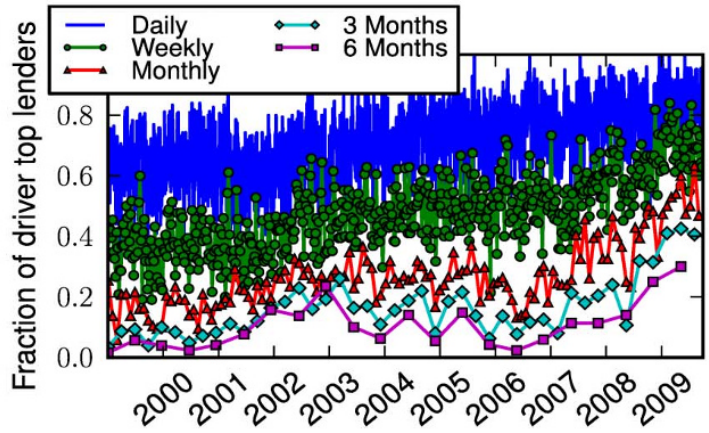

Figure $4 \mid$ (a). Average closeness of drivers (red bars) and average fraction of the aggregate lending accounted for by the driver banks (yellow bars), for the different aggregation time scales. Both values are obtained after averaging over all available network snapshots at the considered time resolution. Maximum closeness is achieved for the monthly network. (b). The fraction of top lender banks which are also drivers of the network: not only the drivers are not hubs, they are not even the larger banks, especially at wider aggregation scales. 
have been proposed to cope with liquidity shocks within interbank markets. Traditionally, policies have been mainly based on liquidity injection through open market operations, but it has been proved that targeted intervention on individual banks could be more effective in guaranteeing and restoring the efficient allocation of $\mathrm{credit}^{28}$. This suggests the need for monitoring the system and keeping track of banks that are systemically relevant from a control perspective.

When the interbank system is not operating properly, many banks are likely to become illiquid, but not necessarily insolvent. Nonconventional liquidity channels can allow the central banks to refinance individually solvent banks not receiving credit or major lenders not providing credit due to the loss of reciprocal trusts among banking institutions.

Here we consider interbank systems as networks of ties among banking institutions. Recent advancements in the science of complex networks provide new tools to investigate their controllability. Specifically, the Minimum Input Theorem ${ }^{21}$ allows one to infer which nodes are essential for control purposes (drivers). By exploiting these tools, our analysis contributes to the identification of systemically relevant banks in a network perspective. The importance of highly connected banks has been highlighted in connection with spillover and contagion effects. We add a new dimension from the point of view of the network controllability and monitoring, identifying the drivers of the banking system. This specification is not equivalent but complementary to previously discussed measures of centrality.

Our contribution is twofold. First we contribute to complex network literature: we represent the market of interbank deposits as a network of credit ties and, for the first time, we study the controllability of a temporal network over time and systematically investigate the relationship which exists between network controllability and the aggregation time scale. Our work sheds new light on the debate on interbank market control. We show that no characteristic scale exists in the decay of the fraction of drivers with the time resolution, implying that no optimal timing for bank supervision can be selected based only on that. Nevertheless, other network statistics, such as the persistence of control configurations, indicate the monthly scale as natural for observing the system.

Secondly, a major result of our analysis is that the banks which are more relevant to the overall state of the credit network are neither the most connected nor the top lenders. This strongly suggests the necessity to rethink the policies based exclusively on the TBTF specification of a systemically important institution. According to recent regulation proposals, the ECB has recognized that allowing recapitalization interventions directly on individual banks is necessary (see the European Commission proposal $\operatorname{COM}(2012) 511$ final, 2012/ 0242 (CNS)). They also highlight that the range of targets has to be broadened to include banks other than the largest, usually TBTF, lenders. Our findings contribute a precise indication by identifying those target banks with the network drivers. Indeed we identify drivers with the agents that are more effective in transmitting the monetary policy of the central bank and preventing complete gridlocks of the banking industry.

While studies about network controllability and resilience have provided new insight, future work should be devoted to combining these complementary aspects into the design of concrete intervention policies for banking regulation.

1. Haldane, A. G. \& May, R. M. Systemic risk in banking ecosystems. Nature 469, 351-5 (2011).

2. Battiston, S., Puliga, M., Kaushik, R., Tasca, P. \& Caldarelli, G. DebtRank: too central to fail? Financial networks, the FED and systemic risk. Sci. Rep. 2, 541 (2012).

3. Schweitzer, F. et al. Economic networks: the new challenges. Science 325, 422-5 (2009).

4. Garlaschelli, D., Battiston, S., Castri, M., Servedio, V. D. P. \& Caldarelli, G. The scale-free topology of market investments. Physica A 350, 2-4 (2003).
5. Galluccio, S., Caldarelli, G., Marsili, M. \& Zhang, Y.-C. Scaling in currency exchange. Physica A 245, 423-436 (1997).

6. Caldarelli, G. Scale-Free Networks (Oxford University Press, Oxford, 2007).

7. Garlaschelli, D., Capocci, A. \& Caldarelli, G. Self-organized network evolution coupled to extremal dynamics. Nat. Phys. 3, 813-817 (2007).

8. De Masi, G., Iori, G. \& Caldarelli, G. Fitness model for the Italian interbank money market. Phys. Rev. E 74, 066112 (2006).

9. Barabási, A.-L. Scale-Free Networks: A Decade and Beyond. Science 325, 412-413 (2009).

10. Barabasi, A.-L. The network takeover. Nat. Phys. 8, 14-16 (2012).

11. Ribeiro, B., Perra, N. \& Baronchelli, A. Quantifying the effect of temporal resolution on time-varying networks (2012). arXiv:1211.7052.

12. Krings, G., Karsai, M., Bernhardsson, S., Blondel, V. D. \& Saramäki, J. Effects of time window size and placement on the structure of an aggregated communication network. EPJ Data Science 1, 4 (2012).

13. Allen, F. \& Gale, D. Financial Contagion. J. Polit. Econ. 108, 1 (2000).

14. Podobnik, B., Horvatic, D., Petersen, A. M., Urosevic, B. \& Stanley, H. E. Bankruptcy risk model and empirical tests. Proc. Natl. Acad. Sci. U.S.A. 107, 18325-30 (2010).

15. Caballero, R. J. Macroeconomics after the Crisis: Time to Deal with the Pretenseof-Knowledge Syndrome. J. Econ. Perspect. 24, 85-102 (2010).

16. Battiston, S., Delli, G. D., Gallegati, M., Greenwald, B. \& Stiglitz, J. E. Liaisons dangereuses: Increasing connectivity, risk sharing, and systemic risk. J. Econ. Dyn. Control 36, 1121-1141 (2012).

17. Stern, G. H. \& Feldman, R. J. Too Big To Fail (Brookings Institution Press, Washington, 2004)

18. Acharya, V. V. \& Merrouche, O. Precautionary Hoarding of Liquidity and InterBank Markets: Evidence from the Sub-prime Crisis. NBER Work. Pap. Ser. No. 16395 (2010).

19. Heider, F., Hoerova, M. \& Holthausen, C. Liquidity Hoarding and Interbank Market Spreads: The Role of Counterparty Risk (2009).

20. Galbiati, M. \& Sorämaki, K. Liquidity-saving mechanisms and bank behaviour. Bank of England Working Paper No. 400 (2010).

21. Liu, Y.-Y., Slotine, J.-J. \& Barabási, A.-L. Controllability of complex networks. Nature 473, 167-73 (2011).

22. Lin, C. T. Structural Controllability. IEEE Trans. Automat. Contr. 19, 201-208 (1974).

23. Schneider, C. M., Moreira, A. A., Andrade, J. S., Havlin, S. \& Herrmann, H. J. Mitigation of malicious attacks on networks. Proc. Natl. Acad. Sci. U.S.A. 108, 3838-41 (2011).

24. Preis, T., Schneider, J. J. \& Stanley, H. E. Switching processes in financial markets Proc. Natl. Acad. Sci. U.S.A. 108, 7674-8 (2011).

25. Mantegna, R. N. \& Stanley, H. E. Scaling behaviour in the dynamics of an economic index. Nature 376, 46-49 (1995).

26. Amaral, L. A. N. et al. Scaling behavior in economics: I. Empirical Results for Company Growth. Journal de Physique I 7, 621-633 (1997).

27. Gopikrishnan, P., Plerou, V., Nunes Amaral, L. A., Meyer, M. \& Stanley, H. E. Scaling of the distribution of fluctuations of financial market indices. Phys. Rev. E 60, 5305-5316 (1999).

28. Freixas, X. Monetary policy in a systemic crisis. Oxford Review of Economic Policy 25, 630-653 (2009)

\section{Acknowledgments}

The authors acknowledge support from FET Open Project FOC "Forecasting Financial Crises" nr. 255987 and Progetto di Interesse CNR "CrisisLab”, and Prof. Giulia Iori of City University in London for fruitful discussions about the topic and the paper. Giampaolo Gabbi acknowledges support from EU Framework Program SSH.2010.1.2-1.

Financialisation, economy, society and sustainable development (FESSUD) and Sda Bocconi for the purchase of the database.

\section{Author contributions}

All authors contributed equally to the analysis of the dataset and to the interpretation of the results of this analysis, both from the point of view of Network Theory as well as in terms of policy implications. They also contributed equally to the writing and reviewing of the manuscript before and after receiving the Reviewers' comments.

\section{Additional information}

Supplementary information accompanies this paper at http://www.nature.com/ scientificreports

Competing financial interests: The authors declare no competing financial interests.

License: This work is licensed under a Creative Commons

Attribution-NonCommercial-NoDerivs 3.0 Unported License. To view a copy of this license, visit http://creativecommons.org/licenses/by-nc-nd/3.0/

How to cite this article: Delpini, D. et al. Evolution of Controllability in Interbank Networks. Sci. Rep. 3, 1626; DOI:10.1038/srep01626 (2013). 